\title{
A Novel Vibration Sensor Based on Bidomain Lithium Niobate Crystal
}

\author{
I.V. Kubasov*, A.M. Kislyuk, M.D. Malinkovich, A.A. Temirov, S.V. Ksenich, \\ D.A. Kiselev, A.S. Bykov and Y.N. Parkhomenko \\ National University of Science and Technology MISIS, Leninskiy prosp. 4, 119049 Moscow, Russia

\begin{abstract}
A novel piezoelectric vibration sensor based on lithium niobate single crystal with bidomain ferroelectric structure was studied. Sensitivity to acceleration of the order of $0.03 \mathrm{~V} / \mathrm{g}\left(g=9.8 \mathrm{~m} / \mathrm{s}^{2}\right.$ is acceleration of gravity) was measured at resonant frequency. Due to high Curie point, weak temperature dependence of piezoelectric coefficients of lithium niobate and absence of glue layer in the construction, the bidomain $\mathrm{LiNbO}_{3}$-based sensor is able to withstand more than $450^{\circ} \mathrm{C}$.
\end{abstract}

DOI: 10.12693/APhysPolA.134.106

PACS/topics: vibration sensor, lithium niobate, bidomain crystal, sensitivity to acceleration.

\section{Introduction}

Crystalline ferroelectric bulk materials are widely used in the production of electromechanical systems, (e.g. vibration sensors, precision actuators and ultrasonic devices) due to their outstanding piezoelectric properties [1-3]. Lead-based ferroelectric materials as PZT $\left(\mathrm{PbZr}_{x} \mathrm{Ti}_{1-x} \mathrm{O}_{3}\right)$ or PMN-PT $\left([1-x] \mathrm{PbMg}_{1 / 3} \mathrm{Nb}_{2 / 3} \mathrm{O}_{3}-\right.$ $\left.[x] \mathrm{PbTiO}_{3}\right)$ are commonly proposed materials for such devices $[4,5]$ due to their very high piezoelectric coefficients, which can be varied in the relatively wide range by changing of material composition. However, the Curie points of PZT and PMN-PT do not exceed $360^{\circ} \mathrm{C}$ and $260^{\circ} \mathrm{C}$, respectively [6]. Thus, high temperature applications are impossible. Moreover, these materials contains lead, which is toxic and harmful for the environment.

Ferroelectric lead-free single crystals such as lithium niobate $\left(\mathrm{LiNbO}_{3}\right)$ and lithium tantalate $\left(\mathrm{LiTaO}_{3}\right)$ have high Curie temperature, demonstrate good thermal and electrical stability and almost do not degrade in time, but have too small piezoelectric coefficients (at least one order smaller than that of PZT ceramics and smaller by two orders than that of PMN-PT relaxor single crystals).

Thus it is inconvenient to utilized these materials in electromechanical piezoelectric devices. One of the ways to solve this problem is to create a bimorph based on two single crystals. However gluing or sintering of crystalline plates nullifies the positive effect of the materials, stable at high temperatures.

Several ways of formation of bidomain ferroelectric structure "head-to-head" and "tail-to-tail" in $\mathrm{LiNbO}_{3}$ and $\mathrm{LiTaO}_{3}$ were proposed previously [7-13]. When electric field is applied, one of domains of bidomain structure expands and the other domain contracts, so the whole crystalline plate bends similar to bimorph principle.

*corresponding author; e-mail: kubasov.ilya@gmail.com
Bidomain crystals demonstrate strongly linear dependence of deformation on the voltage applied to crystal faces $[13,14]$. Capability of bidomain $\mathrm{LiNbO}_{3}$ crystals to predictably transform voltage into deformation and contrariwise, and to withstand high temperatures is suggested to be used in actuators [13-15], energy harvesters [16] and in magnetoelectric laminates [17].

In this study, we used such structure in order to create and investigate a prototype of a lead-free vibration sensor based on lithium niobate, with high sensitivity and, in prospect with wide range of operating temperatures.

\section{Experimental}

Commercially available $y+128^{\circ}$-cut lithium niobate single crystal plates produced by Elan Ltd. (St. Petersburg, Russia) were used in this study. In order to form the ferroelectric bidomain structure in a plate we applied annealing in non-uniform thermal field using a rapid infrared heating-cooling system ULVAC VHC-P610, as was described in [13].

The crystallographic cut of $y+128^{\circ}$ was selected due to its largest transverse piezoelectric coefficient $d_{23}$ among the commercially available $\mathrm{LiNbO}_{3}$ cuts [18]. After annealing, crystals with size of $70 \times 5 \times 0.5 \mathrm{~mm}^{3}$, with long side perpendicular to the $x$ axis were cut from the initial bidomain plate. In order to apply electrodes on polar faces of the bidomain $\mathrm{LiNbO}_{3}$ crystal the DC magnetron sputtering of copper target was used. Finally, the single crystalline bimorph was fastened as a cantilever to an aluminum base using a clamp with four screws.

The bidomain crystal is able to produce voltage when bent, due to direct piezoelectric effect. In order to provide bending with controlled frequency and magnitude we used a PZT tube actuator with a thin metal rod attached to the free end of the crystal, as shown in Fig. 1. Deformations of PZT tube were excited by a signal generator. Despite the simplicity and functionality, such design leads to partial clamping of the free end of the bidomain crystal thus the plate does not operate as a "pure" 
cantilever. At the same time, movements can be provided in a very controlled manner.

Due to high output impedance of the piezoelectric crystal it is necessary to use a preamplifier instead of a direct connection of an oscilloscope to the vibrational sensor prototype. For the study we have assembled a low noise preamp based on differential amplifier AD8221 (Analog Instruments Inc.). The input resistance of the preamp was measured to be as high as $100 \mathrm{M} \Omega$. After the preamplification the voltage produced by the bidomain crystal was registered by dual-channel oscilloscope (Agilent DSO-X 3032A). In order to decrease the electric noise we used shielding of the sensor prototype, of the preamplifier and of all signal wires during the experiment.
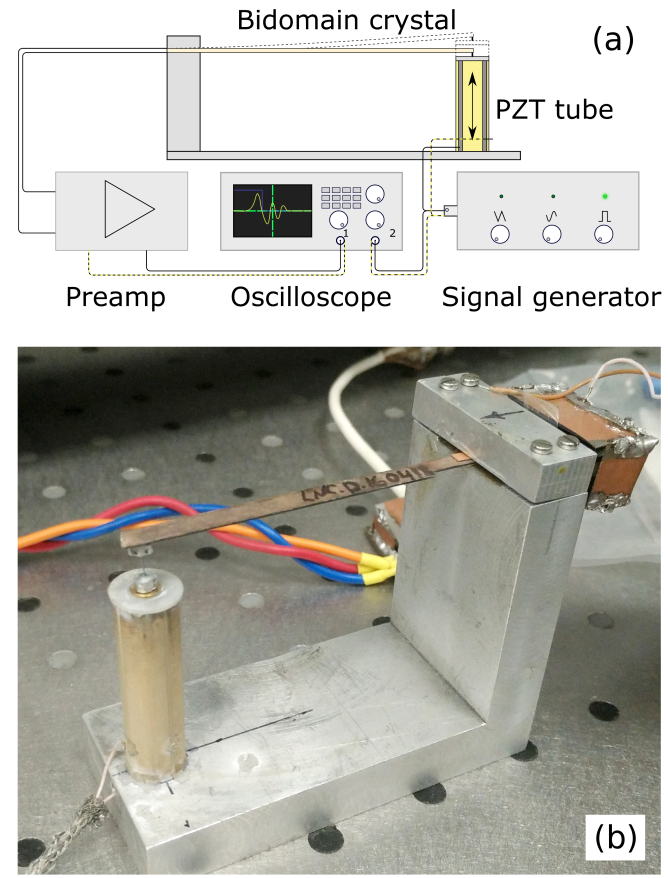

Fig. 1. (a) Schematic representation of the testing equipment; (b) photograph of the vibrational sensor prototype fastened to the base and to PZT tube.

\section{Results and discussion}

Figure 2a demonstrates the output voltage of the sensor prototype when sinusoidal deformation with magnitude of $270 \mathrm{~nm}$ is applied to the free end of the plate. We discovered that $70 \mathrm{~mm}$ long bidomain with thickness of $0.5 \mathrm{~mm}$ produces $\approx 200 \mu \mathrm{V}$ at low frequency vibrations (down to $20 \mathrm{~Hz}$ ) and more than $5 \mathrm{mV}$ at the resonant frequency of $470 \mathrm{~Hz}$ when deflected by PZT tube for $1 \mathrm{~nm}$. This resonant frequency corresponds not to the bidomain crystal only, but to the whole system, which contains crystal, PZT tube, metal rod and the base.

Because the resonant frequency of PZT-tube is much higher than the resonant frequency of bidomain cantilever and due to almost linear dependence of elongation at low voltages, there is a possibility to estimate the
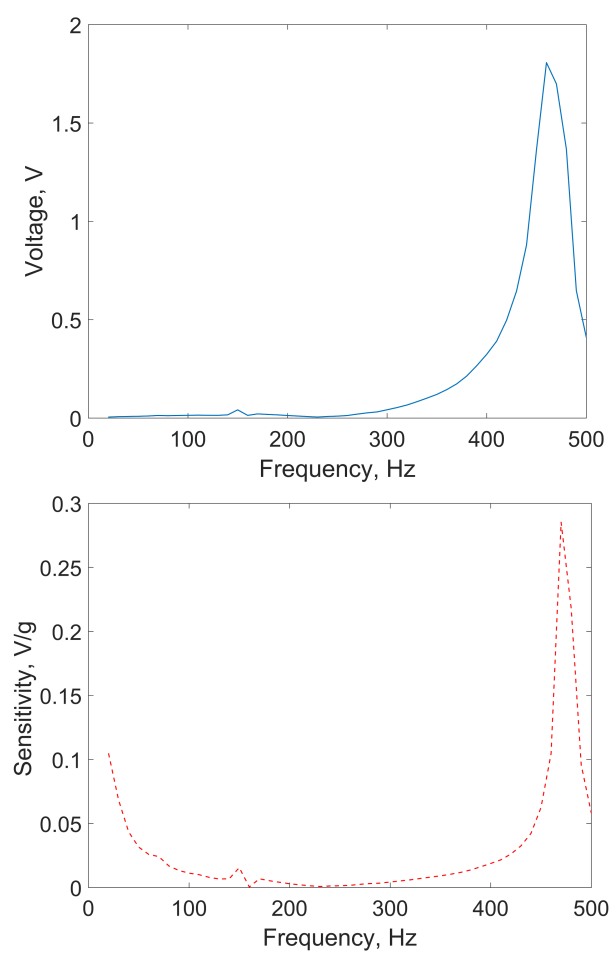

Fig. 2. Dependence of the output voltage (a) and sensitivity (b) on the frequency of the deflection of the free end of the vibrational sensor prototype ( $g$ is acceleration of gravity).

sensitivity of the vibration sensor based on a bidomain crystal. For this purpose we applied a sinusoidal signal to the tube and after two differentiations have calculated the acceleration of the end of bidomain crystal. The dependence of the voltage produced by the sensor prototype on the applied acceleration in terms of $g=9.8 \mathrm{~m} / \mathrm{s}^{2}$ is shown in Fig. 2b.

The highest sensitivity is achieved at the resonant frequency $(0.28 \mathrm{~V} / \mathrm{g})$. However, there is also a region of high sensitivity at low frequencies. These two regions correspond to the situation when the output voltage and the acceleration of the free end of the bidomain crystal are in the same phase.

Anther behavior of device can be noticed within 100 $400 \mathrm{~Hz}$ range. Here one can observe a very small sensitivity despite not very low output voltage. This seems to be due to the destructive addition of two vibrations from different parts of our testing tools. The evidence of it was seen in the oscilloscope signal (Fig. 3). Most clearly this effect appears at frequency of $230 \mathrm{~Hz}$. This might be associated with the construction of the prototype. The end of cantilever is not completely free, so different harmonics can be excited, some of which interfere destructively.

The sensitivity plot of the sensor prototype is very similar to the one obtained for high temperature vibrational sensor proposed by Kim, et al. [19]. The main difference is in frequency range due to different modes used 


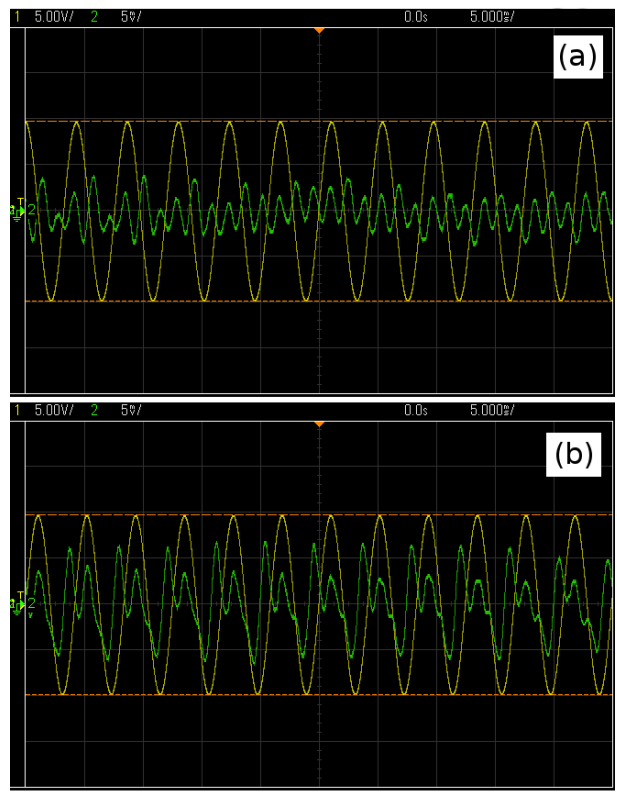

Fig. 3. Output signal from vibrational sensor prototype for $230 \mathrm{~Hz}$ (a) and $240 \mathrm{~Hz}$ (b) excitation frequencies. Curve 1 (yellow color) corresponds to voltage on PZT tube, curve 2 (green color) corresponds to output voltage from preamplifier.

in [19] and in this study. However, the sensitivity of our device is relatively small. This might be caused by usage of the preamplifier with a relatively small input impedance, while Kim, et al. utilized a charge amplifier and a lock-in. Moreover, here we did not add seismic mass at the free end of the cantilever that could also influence the sensitivity.

\section{Conclusions}

In conclusion, a prototype of piezoelectric vibration sensor was designed and tested. Bidomain lithium niobate plate fastened as a cantilever was used as a sensitive element. The highest sensitivity of $0.28 \mathrm{~V} / \mathrm{g}$ was achieved at the resonant frequency of $470 \mathrm{~Hz}$. The effect of sensitivity decrease was observed at frequency of $230 \mathrm{~Hz}$. Possible reason for this decrease is the usage of a construction with partially clamped free end of piezoelectric plate. In the next study we are going to test sensor at high temperature. It is also possible to increase sensitivity of the sensor prototype using seismic mass at the free end and by improving the preamplifier circuit.

\section{Acknowledgments}

The study was supported by the Ministry of Education and Science of the Russian Federation (Federal Targeted Program for Research and Development in Priority Areas of Development of the Russian Scientific and Technological Complex for 2014-2020) (Project ID RFMEFI57816X0187).

\section{References}

[1] E.F. Crawley, J. De Luis, AIAA Journal 25, 1373 (1987).

[2] J.A. Gallego-Juarez, J. Phys. E: Scientific Instr. 22, 804 (1989).

[3] S.S. Rao, M. Sunar, Appl. Mech. Rev. 47, 113 (1994).

[4] P.K. Panda, J. Mater. Sci. 44, 5049 (2009).

[5] I.A. Ivan, M. Rakotondrabe, J. Agnus, R. Bourquin, N. Chaillet, P. Lutz, J.C. Poncot, R. Duffait, O. Bauer, Rev. Adv. Mater. Sci. 24, 1 (2010).

[6] S.E. Park, T.R. Shrout, IEEE Transact. on Ultrasonics, Ferroelectrics, and Freq. Control 44, 1140 (1997).

[7] K. Nakamura, H. Ando, H. Shimizu, Jap. J. Appl. Phys. 26, 198 (1987).

[8] K. Nakamura, H. Shimizu, Ferroelectrics 93, 211 (1989).

[9] I.V. Kubasov, A.M. Kislyuk, A.S. Bykov, M.D. Malinkovich, R.N. Zhukov, D.A. Kiselev, S.V. Ksenich, A.A. Temirov, N.G. Timushkin, Y.N. Parkhomenko, Crystallography Reports 61, 258 (2016).

[10] V.D. Kugel, G. Rosenman, Appl. Phys. Lett. 62, 2902 (1993).

[11] V.V. Antipov, A.S. Bykov, M.D. Malinkovich, Y.N. Parkhomenko, Ferroelectrics 374, 65 (2008).

[12] M.D. Malinkovich, A.S. Bykov, I.V. Kubasov, D.A. Kiselev, S.V. Ksenich, R.N. Zhukov, A.A. Temirov, N.G. Timushkin, Y.N. Parkhomenko, Russian Microelectronics 45, 582 (2016).

[13] A.S. Bykov, S.G. Grigoryan, R.N. Zhukov, D.A. Kiselev, S.V. Ksenich, I.V. Kubasov, M.D. Malinkovich, Y.N. Parkhomenko, Russian Microelectronics 43, 536 (2014).

[14] K. Nakamura, H. Shimizu, Ferroelectrics 93, 211 (1989).

[15] A.E. Blagov, A.S. Bykov, I.V. Kubasov, M.D. Malinkovich, Y.V. Pisarevskii, A.V. Targonskii, I.A. Eliovich, M.V. Kovalchuk, Instrum. Exp. Tech. 59, 728 (2016).

[16] T. Funasaka, M. Furuhata, Y. Hashimoto, K. Nakamura, IEEE Proceedings of Ultrasonics Symposium 1, 959 (1998).

[17] J. Vidal, A.V. Turutin, I.V. Kubasov, M.D. Malinkovich, Y.N. Parkhomenko, S.P. Kobeleva, A.L. Kholkin, N.A. Sobolev, IEEE Transact. on Ultrasonics, Ferroelectrics, and Freq. Control 64, 1102 (2017).

[18] I.V. Kubasov, A.V. Popov, A.S. Bykov, A.A. Temirov, A.M. Kislyuk, R.N. Zhukov, D.A. Kiselev, M.V. Chichkov, M.D. Malinkovich, Yu.N. Parkhomenko, Russian Microelectronics 46 , 557 (2017).

[19] K. Kim, S. Zhang, G. Salazar, X. Jiang, Sens. Actuat. A 178, 40 (2012). 